\title{
ON THE DECOMPOSITION PRINCIPLE AND A PERSSON TYPE THEOREM FOR GENERAL REGULAR DIRICHLET FORMS
}

\author{
DANIEL LENZ ${ }^{1}$ AND PETER STOLLMANN ${ }^{2}$
}

\begin{abstract}
We present a decomposition principle for general regular Dirichlet forms satisfying a spatial local compactness condition. We use the decomposition principle to derive a Persson type theorem for the corresponding Dirichlet forms. In particular our setting covers LaplaceBeltrami operators on Riemannian manifolds, and Dirichlet forms associated to $\alpha$-stable processes in Euclidean space.
\end{abstract}

\section{INTRODUCTION}

Both decomposition principles and Persson's theorem deal with the essential spectrum of suitable Laplace type operators $\mathcal{L}$ acting on a locally compact space $X$. With notation and underlying concepts to be explained in further detail below the decomposition principle asserts

$$
\sigma_{\text {ess }}(\mathcal{L})=\sigma_{\text {ess }}\left(\mathcal{L}_{X \backslash K}\right) .
$$

Here, $\sigma_{\text {ess }}$ denotes the essential spectrum and $\mathcal{L}_{X \backslash K}$ denotes the restriction of $\mathcal{L}$ to the complement of a compact set $K$. On the other hand Persson's theorem states

$$
\inf \sigma_{\text {ess }}(\mathcal{L})=\lim _{K \rightarrow X} \inf \sigma\left(\mathcal{L}_{X \backslash K}\right) .
$$

Here, $\sigma$ denotes the spectrum and the limit is taken along the net of compact subsets of $X$.

Both Persson's theorem and the decomposition principle are concrete instances of the general philosophy that the essential spectrum of Laplacians 'comes from infinity'. Moreover, Persson's theorem can be seen as a rather direct consequence of the decomposition principle. Indeed, the equality asserted in Persson's theorem can be thought of as two inequalities. Now, one of them (viz ' $\geq$ ') immediately follows from the decomposition principle and the other inequality (viz ' $\leq$ ') can be shown rather directly and is, in essence, well-known.

The decomposition principle has been a mainstake in spectral theory for at least a hundred years. Indeed, already Weyl's celebrated 1910 paper [62] on absence of essential spectrum for Schrödinger type operators of the form $-\Delta+V$ with $V(x) \rightarrow \infty, x \rightarrow \infty$, on $L^{2}\left(\mathbb{R}^{d}\right)$ crucially relies on it. These ideas have then been taken up later to even characterize the growth of $V$ yielding absence of essential spectrum in [47, 46] and in [40]. In fact, we also use some ideas and concepts from the latter article.

Date: 22.5 .2017 . 
In 1958 Persson then proved the result named after him for general divergence type Schrödinger operators, [48, actually without using a decomposition principle! His result became an important tool in corresponding considerations in mathematical physics.

In a spirit very similar to Weyl's original approach, Donnelly and Li [16] stated and used a decomposition principle for Riemannian manifolds in their famous work from 1979 showing absence of essential spectrum for LaplaceBeltrami operators whenever the sectional curvature of the underlying manifold tends to $-\infty$.

In the middle of the 90ies the pioneering work of Sturm [60, 61] made it clear that strongly local Dirichlet forms provide a proper setting for and generalization of spectral geometry of Laplacians on Euclidean space and on manifolds. The key ingredient of this approach is the so-called intrinsic metric. To make the theory work one has to assume that this intrinsic metric generates the original topology. (As shown in 19 this assumption can be relaxed to continuity of the intrinsic metric in various circumstances, see [58] as well). Under this compatibility assumption as well as some technical assumptions Grillo 28 proved in 1998 a general Persson type theorem for strongly local Dirichlet forms. Quite remarkably, Grillo's work does not contain a decomposition principle either. We note in passing that various other parts of general spectral theory could be shown for strongly local Dirichlet forms satisfying the compatibility condition in the last ten years [8, 7, 41]. All the works mentioned so far deal with regular strongly local Dirichlet forms (corresponding to Markov processes with continuous paths). Recent years have now seen an ever increasing interest in non-local Dirichlet forms (corresponding to Markov processes with jumps). Here, most prominent cases concern Laplacians on graphs and $\alpha$-stable processes, see Section 4 below for a selection of references on these topics

For Laplacians on graphs with standard weights Persson's theorem can be found in Keller [35] (see as well the earlier work of Fujiwara for related material dealing with the normalized Laplacian [21]). This result was then generalized to weighted locally finite graphs by Keller and Lenz [37]. For these graphs validity of a decomposition principle is quite straightforward and accordingly a Persson type theorem is rather easy to derive. However, so far neither a decomposition principle nor Persson's theorem are known for general graphs let alone more complicated non-local situations such as $\alpha$-stable processes. This is the starting point of the present note.

More specifically, we present a decomposition principle (Section 21) and a Persson type theorem (Section 3) valid for all regular Dirichlet forms satisfying a spatial local compactness condition.

This condition is then shown to be valid in a variety of situations (Section (4). These situations include the already known case of Laplace-Beltrami operators on manifolds. More importantly, they also include rather general graphs as well as $\alpha$-stable processes in Euclidean space. To the best of our knowledge these are the first results giving a decomposition principle and a Persson theorem for jump processes which are not coming from graphs.

All these considerations are given after a first introduction into the topic of Dirichlet forms in Section 1. We finally discuss in Section [5 a generalization 
of our main results to certain Schrödinger type operators. More precisely, we will be able to deal with measure perturbations where the negative part has to fulfill some Kato condition while the positive part is quite arbitrary.

\section{Preliminaries on Dirichlet forms and Selfadjoint operators}

Our investigations are situated in the context of (regular) Dirichlet forms on locally compact separable spaces. In this section we introduce the necessary background and notation (see e.g. [6, 14, 23, 43]). The basic relevance of regular Dirichlet forms in our context comes from the fact that they are in one-to-one correspondence to Markov processes with paths satisfying a regularity condition known as càdlàg. Thus, Dirichlet forms provide an analytic tool to work with Markov processes.

Throughout we let $X$ be a locally compact, separable metric space and $m$ a positive Radon measure on $\mathrm{X}$ with $\operatorname{supp} m=X$. We will only consider real valued function on $X$. (Of course, complex valued functions could easily be considered as well after complexifying the corresponding Hilbert spaces and forms.) By $C_{c}(X)$ we denote the set of continuous functions on $X$ with compact support and by $C_{0}(X)$ the closure of $C_{c}(X)$ with respect to the supremum norm. The space $L^{2}(X, m)$ is the space of classes of measurable and (with respect to $m$ ) square integrable real valued functions. The norm on $L^{2}(X, m)$ will be denoted by $\|\cdot\|_{L^{2}(X, m)}$ or, if the space is clear from the context, just by $\|\cdot\|$. The space $L^{\infty}(X, m)$ is the space of all classes of essentially bounded functions.

The characteristic function of a set $M$ will be denoted by $\mathbb{1}_{M}$ i.e. $\mathbb{1}_{M}(x)=1$ if $x \in M$ and $\mathbb{1}_{M}(x)=0$ else.

A closed non-negative form on $L^{2}(X, m)$ consists of a dense subspace $\mathcal{D} \subset$ $L^{2}(X, m)$ and a sesquilinear map $\mathcal{E}: \mathcal{D} \times \mathcal{D} \rightarrow \mathbb{R}$ with $\mathcal{E}(f, f) \geq 0$ for all $f \in \mathcal{D}$ such that $\mathcal{D}$ is complete with respect to the energy norm $\|\cdot\|_{\mathcal{E}}$ defined by

$$
\|u\|_{\mathcal{E}}:=\left(\mathcal{E}(u, u)+\|u\|_{L^{2}(X, m)}^{2}\right)^{1 / 2} .
$$

Whenever $\mathcal{E}$ is a closed form there exists a unique selfadjoint operator $\mathcal{L}$ on $L^{2}(X, m)$ with

$$
\langle\mathcal{L} u, v\rangle=\mathcal{E}(u, v)
$$

for all $u$ in the domain of $\mathcal{L}$ and all $v \in \mathcal{D}$. This operator $\mathcal{L}$ is non-negative. A closed form is said to be a Dirichlet form if for any $u \in \mathcal{D}$ and any normal contraction $C: \mathbb{R} \rightarrow \mathbb{R}$ we have also

$$
C \circ u \in \mathcal{D} \text { and } \mathcal{E}(C \circ u) \leq \mathcal{E}(u) \text {. }
$$

Here, $C: \mathbb{R} \rightarrow \mathbb{R}$ is called a normal contraction if $C(0)=0$ and $\mid C(\xi)-$ $C(\zeta)|\leqslant| \xi-\zeta \mid$ for any $\xi, \zeta \in \mathbb{R}$. Typical examples of normal contractions are modulus and positive and negative part of a number. A Dirichlet form is called regular if $\mathcal{D} \cap C_{c}(X)$ is dense both in $\left(\mathcal{D},\|\cdot\|_{\|\cdot\|_{\mathcal{E}}}\right)$ and $\left(C_{c}(X),\|\cdot\|_{\infty}\right)$. By the fundamental connection to probability theory, see Fukushima's classic [22], to every regular Dirichlet form there exists an associated Markov process $\left(\Omega,\left(\mathbb{P}^{x}\right)_{x \in X},\left(X_{t}\right)_{t \geqslant 0}\right)$ with state space $X \cup\{\infty\}$ related to the form via the semigroup:

$$
e^{-t \mathcal{L}} f(x)=\mathbb{E}^{x}\left[f \circ X_{t}\right] \text { a.e. }
$$


for any $f \in L^{2}(X)$ and $t \geqslant 0$. A regular Dirichlet $\mathcal{E}$ form is said to be local if $\mathcal{E}(f, g)=0$ whenever $f$ and $g$ have disjoint support. If even $\mathcal{E}(f, g)=0$ whenever $g$ is constant on a neighborhood of the support of $f$ then $\mathcal{E}$ is said to be strongly local. Typical examples of operators associated to strongly local Dirichlet forms are Laplace-Beltrami operatators on manifolds. Typical example of operators associated to non-local Dirichlet forms are Laplacians on graphs and fractional Laplacians which are the generators of $\alpha$-stable processes.

We will need the restrictions to open sets of operators associated to a Dirichlet form. Let $\mathcal{L}$ be the selfadjoint operator on $L^{2}(X, m)$ associated to the regular Dirichlet form $\mathcal{E}$ with domain $\mathcal{D}$. Let $U$ be an open subset of $X$. Then, the restriction of $\mathcal{E}$ to

$$
\overline{\mathcal{D} \cap C_{c}(U)}\|\cdot\|_{\mathcal{E}}
$$

is a regular Dirichlet form on $L^{2}(U, m)$ (this is easy to see, Theorem 4.4.2 from [22] contains it). This form will be denoted by $\mathcal{E}_{U}$. The selfadjoint operator associated to $\mathcal{E}_{U}$ will be denoted by $\mathcal{L}_{U}$.

Using the hitting time of $B:=X \backslash U$

$$
\sigma_{B}:=\inf \left\{t \geqslant 0 \mid X_{t} \in B\right\}
$$

we obtain the following instance of the Feynman-Kac formula that allows for a probabilistic interpretation of the semigroup generated by $\mathcal{L}_{U}$ :

$$
e^{-t \mathcal{L}_{U}} f(x)=\mathbb{E}^{x}\left[f \circ X_{t} \cdot \mathbb{1}_{\left\{\omega \mid \sigma_{B}(\omega)>t\right\}}\right] \text { a.e. }
$$

for $f \in L^{2}(U)$ and $t \geqslant 0$; see [22], Section 4.1 and Thm 4.4.2, p. 111

By construction the operator $\mathcal{L}_{U}$ acts in $L^{2}(U, m)$. In order to compare the operators $\mathcal{L}$ and $\mathcal{L}_{U}$ and their spectral properties we will have to extend functions of $\mathcal{L}_{U}$ from operators on $L^{2}(U)$ to operators on $L^{2}(X)$. We will do so by extending $\varphi\left(\mathcal{L}_{U}\right)$ by 0 on $L^{2}(X \backslash U, m)$ for $\varphi \in C_{0}(\mathbb{R})$. This extension by 0 is well in line with the idea that $\mathcal{L}_{U}$ arises from $\mathcal{L}$ by adding a potential with value $\infty$ on $X \backslash U$ which would mean that $\mathcal{L}_{U}=\infty$ on $L^{2}(X \backslash U, m)$. For our later considerations the extension of $e^{-t \mathcal{L}_{U}}$ has the advantage that in this way (1) is valid for all $f \in L^{2}(X)$. This extension will be done tacitly in the sequel; however, in the proof of Theorem 2.3 we have to be a little more careful in one step.

Further, we will need some notions from potential theory of Dirichlet forms and use the connection to stochastic processes. The capacity is a set function associated to a Dirichlet form. It measures the size of sets adapted to the form. It is defined as follows: For $U \subset X, U$ open, we define

$$
\operatorname{cap}(U):=\inf \left\{\left(\mathcal{E}(v, v)+\|v\|_{L^{2}(X, m)}^{2}\right)^{1 / 2}: v \in \mathcal{D}, \mathbb{1}_{U} \leqslant v\right\},
$$

with the usual convention that $\inf \emptyset=\infty$. For arbitrary $A \subset X$, we then set

$$
\operatorname{cap}(A):=\inf \{\operatorname{cap}(U): A \subset U\}
$$

(see [23], Section 2.1). For regular forms the capacity of relatively compact sets can easily be seen to be finite. The 1-equilibrium potential $e_{B}$ of a set of finite capacity can be thought of as the minimizer in the variational definition of the capacity given above, see [23], Theorem 2.1.5. It has an intimate 
relation to the first hitting time of the Markov process $\left(\Omega,\left(\mathbb{P}^{x}\right)_{x \in X},\left(X_{t}\right)_{t \geqslant 0}\right)$ corresponding to $(\mathcal{E}, \mathcal{D})$ viz

$$
e_{B}(x)=\mathbb{E}^{x}\left[e^{-\sigma_{B}}\right]
$$

for $m$-a.e. $x \in X$ by [22], formula (4.2.9) on p. 99 and Thm 4.3.5, p. 106.

The spectrum of a selfadjoint operator $A$ will be denoted by $\sigma(A)$ i.e. $\sigma(A)$ is the set of all real numbers $\lambda$ such that $A-\lambda I$ is not continuously invertible (where $I$ denotes the identity). The spectrum of $A$ can be decomposed in two disjoint parts viz the set of eigenvalues of finite multiplicity and the remaining part. This remaining part is known as the essential spectrum of $A$ and denoted by $\sigma_{e s s}(A)$. Its characteristic feature is that it is stable under compact perturbations, a celebrated fact going back to Weyl, [62, 63].

\section{A general DECOMPosition PRINCIPLE}

In this section we will present a decomposition principle for regular Dirichlet forms. This will give that the essential spectrum of Dirichlet forms is stable under suitable restrictions to complements of compact sets. Throughout the remainder of this section we let a regular Dirichlet form $\mathcal{E}$ on $L^{2}(X, m)$ with domain $\mathcal{D}$ and associated selfadjoint operator $\mathcal{L}$ be given. The crucial assumption will be the following, taken from [40].

Definition 2.1. We say that $\mathcal{L}$ is spatially locally compact if $\mathbb{1}_{E} e^{-\mathcal{L}}$ is a compact operator for every measurable $E$ with $m(E)<\infty$.

Here and in the sequel we identify the function $\mathbb{1}_{E}$ with the corresponding bounded multiplication operator on $L^{2}(X)$.

Remark 2.2. (cf. [40], Remark 2.7)

(a) By [40], Thm 1.3, we could also use the resolvent or spectral projections to characterize spatial local compactness.

(b) As follows from Theorem 1.3 of [40, spatial local compactness is equivalent to compactness of the map $\mathbb{1}_{E}:\left(\mathcal{D},\|\cdot\|_{\mathcal{E}}\right) \longrightarrow L^{2}(X)$ for all measurable $E$ in $X$ with $m(E)<\infty$. In particular, spatial local compactness of $\mathcal{L}$ implies spatial local compactness for all $\mathcal{L}^{\prime}$ such that the form domain of $\mathcal{L}^{\prime}$ embeds into $\mathcal{D}$.

(c) If the semigroup $e^{-t \mathcal{L}}$ is ultracontractive, i.e. $e^{-t \mathcal{L}}: L^{2} \rightarrow L^{\infty}$ for some $t>0$, then $\mathcal{L}$ is spatially locally compact: In fact $\mathbb{1}_{E} e^{-t \mathcal{L}}$ factors through $L^{\infty}$ and the little Grothendieck theorem gives that it is a Hilbert-Schmidt operator, in particular compact. See the discussion in [55], or [15] for the case of positivity preserving semigroups. This is what makes our results applicable to large classes of manifolds, see the discussion in subsection 4.1 below for more details.

(d) Note that $e^{-t \mathcal{L}}$ maps into $L^{\infty}$ whenever there exists an $\alpha>0$ with $D\left(\mathcal{L}^{\alpha}\right) \subset L^{\infty}$. Therefore, the spatial local compactness of $\mathcal{L}$ for $X$ being Euclidean space or a manifold can sometimes easily be checked in terms of compactness of Sobolev embeddings, i.e. in variants of Rellich's theorem [33, Theorem V.4.4.

(e) The Laplacian on quantum or metric graphs is spatially locally compact under quite general assumptions, since its domain is continuously embedded in $L^{\infty}$, see [39]. 
(f) For combinatorial graphs, the condition of spatial local compactness is trivially satisfied, as $\mathbb{1}_{E}$ has finite rank in this case. Therefore we get a rather easy and not very subtle criterion in that case, see below for a discussion of more general graphs.

Here comes the main result of this section.

Theorem 2.3 (Decomposition principle). Let $\mathcal{E}$ be a regular Dirichlet form on $L^{2}(X, m)$ with associated selfadjoint operator $\mathcal{L}$ that is spatially locally compact. Let $B \subset X$ be a closed set of finite capacity and denote $G:=$ $X \backslash B$. Then, the operator $\varphi\left(\mathcal{L}_{G}\right)-\varphi(\mathcal{L})$ is compact for every $\varphi \in C_{0}(\mathbb{R})$. In particular,

holds.

$$
\sigma_{\text {ess }}\left(\mathcal{L}_{G}\right)=\sigma_{\text {ess }}(\mathcal{L})
$$

The remainder of this section is devoted to a proof of this decomposition principle. In fact, we will start with a result that says that the boundary condition that defines $e^{-\mathcal{L}_{G}}$ is not felt too much near infinity.

Proposition 2.4. Let $\mathcal{E}$ be a regular Dirichlet form on $L^{2}(X, m)$ with associated selfadjoint operator $\mathcal{L}$. Let $B \subset X$ be a closed set of finite capacity and denote $G:=X \backslash B$. Then, for measurable $A \subset X$ and $t>0$ we have

$$
\begin{aligned}
\left\|\mathbb{1}_{A}\left(e^{-t \mathcal{L}}-e^{-t \mathcal{L}_{G}}\right)\right\| & \leqslant \sup _{x \in A}\left[\mathbb{P}^{x}\left\{\omega \mid \sigma_{B}(\omega) \leqslant t\right\}\right]^{\frac{1}{2}} \\
& \leqslant e^{\frac{t}{2}} \sup _{x \in A}\left[e_{B}(x)\right]^{\frac{1}{2}}
\end{aligned}
$$

So, the fact that the Dirichlet boundary condition on $B$ is "not felt too much" is measured in terms of the operator norm, while "near infinity" means on sets $A$ far away from $B$ in the sense that the probability of hitting $B$ during the time interval $[0, t]$ when starting in $A$ is small.

This proposition contains one of main new ingredients of our method of proof even though analogous calculations involving the Feynman-Kac formula and Cauchy-Schwarz have been around for quite some time. See, e.g., the proof of Theorem B.1.1 in [53].

Proof. The proof uses an argument from [55] involving the Feynman-Kac formula, (11) above, by which we get that, for any $f \in L^{2}$ and a.e. $x \in X$ :

$$
\begin{aligned}
\left|\left(e^{-t \mathcal{L}}-e^{-t \mathcal{L}_{G}}\right) f(x)\right| & =\left|\mathbb{E}^{x}\left[f \circ X_{t}\left(1-\mathbb{1}_{\left\{\omega \mid \sigma_{B}(\omega)>t\right.}\right)\right]\right| \\
& =\left|\mathbb{E}^{x}\left[f \circ X_{t} \mathbb{1}_{\left.\left\{\omega \mid \sigma_{B}(\omega) \leqslant t\right\}\right]}\right]\right| \\
& \leqslant \mathbb{E}^{x}\left[|f|^{2} \circ X_{t}\right]^{\frac{1}{2}} \mathbb{P}^{x}\left\{\omega \mid \sigma_{B}(\omega) \leqslant t\right\}^{\frac{1}{2}}
\end{aligned}
$$

by Cauchy-Schwarz. Note that the integral appearing in the first factor gives

$$
\mathbb{E}^{x}\left[|f|^{2} \circ X_{t}\right]=e^{-t \mathcal{L}}\left(|f|^{2}\right)(x)
$$

an integrable function, with integral bounded by $\|f\|_{2}^{2}$ as the semigroup of a Dirichlet form is sub-Markovian (and, hence, induces an operator from $L^{1}$ 
to $L^{1}$ with norm not exceeding 1 ). For the second factor, we have, by (2) above

$$
\begin{aligned}
\mathbb{P}^{x}\left\{\omega \mid \sigma_{B}(\omega) \leqslant t\right\} & \leqslant e^{t} \mathbb{E}^{x}\left[e^{-\sigma_{B}}\right] \\
& =e^{t} e_{B}(x),
\end{aligned}
$$

where $e_{B}$ is the one-equilibrium potential. Putting these ingredients together we get

$$
\begin{aligned}
\left\|\mathbb{1}_{A}\left(e^{-t \mathcal{L}}-e^{-t \mathcal{L}_{G}}\right) f\right\|^{2} & =\int_{A}\left|\left(e^{-t \mathcal{L}}-e^{-t \mathcal{L}_{G}}\right) f(x)\right|^{2} d m(x) \\
& \leqslant \int_{A} e^{-t \mathcal{L}}\left(|f|^{2}\right)(x) \cdot \mathbb{P}^{x}\left\{\omega \mid \sigma_{B}(\omega) \leqslant t\right\} d m(x) \\
& \leqslant \sup _{x \in A} \mathbb{P}^{x}\left\{\omega \mid \sigma_{B}(\omega) \leqslant t\right\} \int_{A} e^{-t \mathcal{L}}\left(|f|^{2}\right)(x) d m(x) \\
& \leq \sup _{x \in A} \mathbb{P}^{x}\left\{\omega \mid \sigma_{B}(\omega) \leqslant t\right\}\|f\|^{2} \\
& \leq e^{t} \sup _{x \in A} e_{B}(x)\|f\|^{2},
\end{aligned}
$$

which gives the assertion.

Corollary 2.5. Let $\mathcal{E}$ be a regular Dirichlet form on $L^{2}(X, m)$ with associated selfadjoint operator $\mathcal{L}$. Let $B \subset X$ be a closed set of finite capacity and denote $G:=X \backslash B$. Then there is a sequence $\left(M_{n}\right)_{n \in \mathbb{N}}$ of sets of finite measure such that for every $t>0$

$$
e^{-t \mathcal{L}}-e^{-t \mathcal{L}_{G}}=\|\cdot\|-\lim _{n \rightarrow \infty} \mathbb{1}_{M_{n}}\left(e^{-t \mathcal{L}}-e^{-t \mathcal{L}_{G}}\right)
$$

Proof. We let

$$
M_{n}:=\left\{x \in X \mid e_{B}(x)>\frac{1}{n}\right\}
$$

for a measurable realization of $e_{B}$. As $e_{B}$ is square integrable, $M_{n}$ has finite measure.

Using the preceding Proposition for $A_{n}:=X \backslash M_{n}$ we get the assertion.

Proof of Theorem 2.3. Let $A_{\text {comp }}$ be the set of those functions $\varphi \in C_{0}(\mathbb{R})$ for which $\varphi(\mathcal{L})-\varphi\left(\mathcal{L}_{G}\right)$ is compact.

1st Step: Using the preceding corollary and the assumption that $\mathcal{L}$ is spatially locally compact, we will see that $\exp (-\cdot) \in A_{\text {comp }}$.

In fact, the assumption on $\mathcal{L}$ guarantees that $\mathbb{1}_{M_{n}} e^{-t \mathcal{L}}$ is compact for every $n \in \mathbb{N}$. Since $\mathcal{L}_{G} \geqslant \mathcal{L}$, Corollary 1.5 from [40] gives that $\mathbb{1}_{M_{n}}$ is also $\mathcal{L}_{G}$-relatively compact, i.e., $\mathbb{1}_{M_{n}} e^{-t \mathcal{L}_{G}}$ is compact for every $n \in \mathbb{N}$. Hence, $\mathbb{1}_{M_{n}}\left(e^{-t \mathcal{L}}-e^{-t \mathcal{L}_{G}}\right)$ is compact and so is its limit $e^{-t \mathcal{L}}-e^{-t \mathcal{L}_{G}}$, by $\left(\mathbb{L}_{1}\right)$ above. 2nd Step: $A_{\text {comp }}=C_{0}(\mathbb{R})$, i.e. $\varphi\left(\mathcal{L}_{G}\right)-\varphi(\mathcal{L})$ is compact for every $\varphi \in C_{0}(\mathbb{R})$.

We use a Stone-Weierstraß argument, more precisely [49], Theorem IV.9 and first record that we can ignore the left halfline since both $\mathcal{L}$ and $\mathcal{L}_{G}$ are nonnegative operators. Clearly, $A_{\text {comp }}$ is closed with respect to uniform convergence. By what we checked in the Step 1 , we already know that $A_{\text {comp }}$ separates the points and that for every $x_{0} \in \mathbb{R}$ there is a $\varphi \in A_{\text {comp }}$ that is 
nonzero at $x_{0}$. It remains to show that $A_{\text {comp }}$ is an algebra. Clearly, $A_{\text {comp }}$ is a vector space. For $\varphi, \psi \in A_{\text {comp }}$ we see that

$$
(\varphi \cdot \psi)(\mathcal{L})-(\varphi \cdot \psi)\left(\mathcal{L}_{G}\right)=\varphi(\mathcal{L})\left(\psi(\mathcal{L})-\psi\left(\mathcal{L}_{G}\right)\right)+\left(\varphi(\mathcal{L})-\varphi\left(\mathcal{L}_{G}\right)\right) \psi\left(\mathcal{L}_{G}\right)
$$

is compact and this finishes the second step.

3rd Step: The 2nd Step shows the first claim of the theorem. Given this, the 'In particular' statement of the theorem then follows as the essential spectrum is stable under compact perturbations. This stability can essentially be found e.g. in [51], Theorem XIII.14. However, $\mathcal{L}$ and $\mathcal{L}_{G}$ live on different Hilbert spaces so that the latter result cannot be applied verbatim. At the risk of appearing pedantic, we include the complete argument:

By [51, Lemma 2 from Section XIII.4, called the strong spectral mapping theorem, we infer that

$$
\sigma_{\text {ess }}(\mathcal{L})=\left\{\frac{1}{\lambda}-1 \mid \lambda \in \sigma_{\text {ess }}\left((\mathcal{L}+1)^{-1}\right) \backslash\{0\}\right\},
$$

as well as the respective formula for $\sigma_{e s s}\left(\mathcal{L}_{G}\right)$. Weyl's classical result on stability of the essential spectrum under compact perturbations, see 63] as well as the discussion in [51], Section XIII.4, gives that

$$
\sigma_{\text {ess }}\left((\mathcal{L}+1)^{-1}\right)=\sigma_{\text {ess }}\left(\left(\mathcal{L}_{G}+1\right)^{-1} \oplus 0\right),
$$

where the latter denotes the canonical extension of $\left(\mathcal{L}_{G}+1\right)^{-1}$ (which is defined as a bounded operator on $\left.L^{2}(G, m)\right)$ to all of $L^{2}(X, m)$. It follows that

$$
\sigma_{\text {ess }}\left((\mathcal{L}+1)^{-1}\right) \backslash\{0\}=\sigma_{\text {ess }}\left(\left(\mathcal{L}_{G}+1\right)^{-1}\right) \backslash\{0\},
$$

and the above strong spectral mapping result then gives the claim.

\section{A Persson type theorem}

In this section we present a Persson type theorem for general regular Dirichlet forms. As discussed in the introduction such a theorem is a rather direct consequence of a decomposition principle.

We first recall the following general lower bound on the essential spectrum. The result is well-known and variants have been used in several places, see e.g. [?, 21, 60]. In the form given here it can be found in the recent work [29].

Lemma 3.1. Let $\mathcal{E}$ be a regular Dirichlet form on $L^{2}(X, m)$. If $\left(f_{n}\right)_{n \in \mathbb{N}}$ is a sequence in $\mathcal{D}$ with $\left\|f_{n}\right\|=1$ for all $n \in \mathbb{N}$ and $f_{n} \rightarrow 0$ weakly in $L^{2}(X, m)$ and $\lambda=\lim _{n \rightarrow \infty} \mathcal{E}\left(f_{n}, f_{n}\right)$. Then,

$$
\inf \sigma_{\text {ess }}(\mathcal{L}) \leq \lambda .
$$

Whenever $X$ is a locally compact Hausdorff space, the family of all compact subsets of $X$ forms a net. We will need to take limits along this net. Such limits will be written as $\lim _{K \rightarrow X}$.

Theorem 3.2 (Persson's theorem). Let $\mathcal{E}$ be a regular Dirichlet form on $L^{2}(X, m)$ with domain $\mathcal{D}$ and associated selfadjoint operator $\mathcal{L}$ that is spatially locally compact. Then,

$$
\inf \sigma_{e s s}(\mathcal{L})=\lim _{K \rightarrow X} \inf \sigma\left(\mathcal{L}_{X \backslash K}\right) .
$$


Proof. The infimum of the spectrum is characterized by the variational principle via

$$
\inf \sigma\left(\mathcal{L}_{U}\right)=\inf \left\{\mathcal{E}_{U}(f, f): f \in \mathcal{D}\left(\mathcal{E}_{U}\right),\|f\|=1\right\}
$$

for any open subset $U \subset X$. This easily gives the monotonicity property

$$
\inf \sigma\left(\mathcal{L}_{X \backslash K_{1}}\right) \leq \inf \sigma\left(\mathcal{L}_{X \backslash K_{2}}\right)
$$

whenever $K_{1}, K_{2}$ are compact with $K_{1} \subset K_{2}$. This in turn implies that the limit in question exists (with possible value $\infty$ ) and actually equals

$$
\sup _{K} \inf \sigma\left(\mathcal{L}_{X \backslash K}\right)
$$

where the supremum is taken over all compact $K \subset X$. We will call this limit $\lambda_{0}$. We will now show two inequalities.

$$
\inf \sigma_{\text {ess }}(\mathcal{L}) \leq \lambda_{0}
$$

This follows from the previous lemma. Indeed, we can choose a sequence $\left(K_{n}\right)_{n \in \mathbb{N}}$ of compact subsets of $X$ with $\lambda_{0}=\lim _{n \rightarrow \infty} \inf \sigma\left(\mathcal{L}_{X \backslash K_{n}}\right)$. It follows from the discussion at the beginning of the proof and, in particular, the monotonicity property that we can assume without loss of generality $K_{n} \subset$ $K_{n+1}$ for all $n$ as well as $X=\bigcup_{n} K_{n}$. By definition of the restriction of $\mathcal{L}$ to the complement of a compact set and the variational principle, we can then pick a sequence of functions $f_{n} \in C_{c}\left(X \backslash K_{n}\right) \cap \mathcal{D}$ with $\left\|f_{n}\right\|_{L^{2}(X, m)}=1$ and

$$
\left|\inf \sigma\left(\mathcal{L}_{X \backslash K_{n}}\right)-\mathcal{E}\left(f_{n}, f_{n}\right)\right| \leq \frac{1}{n}
$$

for all $n \in \mathbb{N}$. Then, the $f_{n}$ vanish on $K_{n}, n \in \mathbb{N}$, and hence converge weakly to 0 by our assumptions on the $K_{n}$. Moreover, by construction $\lambda_{0}=$ $\lim _{n \rightarrow \infty} \mathcal{E}\left(f_{n}, f_{n}\right)$. Now, the previous lemma gives the desired inequality.

$\inf \sigma_{e s s}(\mathcal{L}) \geq \lambda_{0}$.

The decomposition principle gives $\inf \sigma_{\text {ess }}\left(\mathcal{L}_{X \backslash K}\right)=\inf \sigma_{\text {ess }}\left(\mathcal{L}_{X}\right)$. Given this, the desired statement follows from the (obvious) inequality

$$
\inf \sigma\left(\mathcal{L}_{X \backslash K}\right) \leq \inf \sigma_{e s s}\left(\mathcal{L}_{X \backslash K}\right) .
$$

Putting together the preceding two inequalities finishes the proof of the theorem.

Remark 3.3. (a) The theorem deals with regular Dirichlet forms provided they are spatially locally compact. In particular the theorem covers Laplace Beltrami operators on manifolds, Laplacians on rather general graphs and $\alpha$-stable processes. Details are discussed in the next section.

(b) The limit over the compact subsets could be replaced by a limit over arbitrary measurable relatively compact subsets (as can be seen from simple monotonicity arguments). 
(c) The limit appearing in the theorem can be expressed in various ways. Among them we mention the following equalities:

$$
\begin{aligned}
& \lim _{K \rightarrow X} \quad \inf \sigma\left(\mathcal{L}_{X \backslash K}\right) \\
& =\quad \sup _{K \subset X \operatorname{cpt}} \inf \sigma\left(\mathcal{L}_{X \backslash K}\right) \\
& =\quad \sup _{K \subset X \operatorname{cpt}} \inf \left\{\frac{\mathcal{E}(f, f)}{\|f\|^{2}}: f \in \mathcal{D}(X \backslash K), f \neq 0\right\} \\
& =\quad \sup _{K \subset X \operatorname{cpt}} \inf \left\{\frac{\mathcal{E}(f, f)}{\|f\|^{2}}: f \in \mathcal{D} \cap C_{c}(X \backslash K), f \neq 0\right\} .
\end{aligned}
$$

Here, the first equality holds by simple monotonicity arguments (as discussed in the proof of the theorem), the second equality is just the Rayleigh-Ritz variation principle and the last equality follows easily from $\mathcal{D}(X \backslash K)=\overline{\mathcal{D} \cap C_{c}(X \backslash K)} \|^{\|\cdot\|_{\mathcal{E}}}$ (which in turn was discussed above).

\section{The RANGe of APPLiCATions - Classes of EXAMPLES}

We now present three classes of examples satisfying the considerations of the preceding sections.

4.1. Regular Dirichlet forms on manifolds. As mentioned in Remark 2.2 (c), the assumption of spatial local compactness is satisfied provided the corresponding heat semigroup is ultracontractive. In particular spatial local compactness holds for the Laplacian on Euclidean space and for LaplaceBeltrami operators on large classes of Riemannian manifolds, so that Theorems 2.3 and 3.2 apply.

In this way we recover the corresponding decomposition prinicple of Donnelly and Li [16] and corresponding instances of Persson's theorem of Grillo [28]. As mentioned above, Grillo's work does not contain a decomposition principle and the method of proof is limited to local forms. Although not stated explicitly, a sort of local compactness is needed, in that results from [5] are used. In that latter paper, the authors assume volume doubling and a Poincaré inequality. This, in turn, implies ultracontractivity, and, moreover, very precise pointwise estimates on Greens functions as shown in [4]. So the compactness properties used in [28] are much stronger than the one we assume in our main theorems.

Let us now discuss the case of manifolds a little more detailed and note first, that we can tacitly assume that we are dealing with a non-compact Riemannian manifold, as otherwise the appearing essential spectra are empty, anyway. We will always consider complete and connected weighted manifolds $(M, \mathbf{g}, \mu)$ as in [25]; the corresponding Laplacian $\Delta_{\mu}$ is defined as in Section 4.2 of the latter reference and its negative, $-\Delta_{\mu}$, generates a regular Dirichlet form, the reader is referred to 25] again for a thorough discussion of this little more general Laplace-Beltrami operator together with the necessary background from Riemannian geometry. A detailed account of heat kernel estimates and the underlying geometric properties can also be found in [24, to which we refer the reader instead of recording the relevant material here. 
Let us first note that ultracontractivity does not hold on any manifold; see e.g., Example 9.17, p. 255 in [25]. It is true for the following classes:

- Cartan-Hadamard manifolds, defined by the property that there sectional curvature $K(p)$ is non-positive. This is the class of manifolds considered in the work of Donnelly and Li, [16]; see [24], Section 7.4 for ultracontractivity in this case.

- Minimal submanifolds of $\mathbb{R}^{N}$; see [24], Section 7.3 for ultracontractivity in this case.

- Manifolds of bounded geometry, defined by a uniform lower bound on Ricci curvature together with a positive uniform lower bound on the injectivity radius; see [24], Section 7.5.

Summarizing these instances we get

Corollary 4.1. Assume that $(M, \mathbf{g}, \mu)$ belongs to the above mentioned classes and let $\Delta$ be the associated Laplacian. Then

(1) The heat semigroup is ultracontractive, in particular $-\Delta$ is spatially locally compact.

(2) For every compact subset $K \subset M$ :

$$
\sigma_{e s s}(-\Delta)=\sigma_{e s s}\left(-\Delta_{M \backslash K}\right)
$$

(3) Perssons' theorem holds:

$$
\inf \sigma_{e s s}(-\Delta)=\sup _{K} \inf \sigma\left(-\Delta_{M \backslash K}\right),
$$

where the supremum is taken over the net of compact subsets of $M$.

The case of manifolds with bounded geometry is a generalization of the case of non-negative Ricci curvature where heat kernel bounds go back to the celebrated work of $\mathrm{Li}$ and Yau, [42]. Far reaching generalizations have been obtained in [52] where uniform lower bounds are replaced by integral bounds. Using our results and the fact that sectional curvature controls isoperimetry and this gives, in turn, lower bounds on the spectrum of the Laplacian just as in [16], we get the following generalization of the result of Donnelly and Li from the latter reference:

Corollary 4.2. Assume that $(M, \mathbf{g}, \mu)$ belongs to the above mentioned classes and that sectional curvature satisfies: $K(p) \rightarrow-\infty$ for $p \rightarrow \infty$, then

$$
\sigma_{\text {ess }}(-\Delta)=\emptyset \text {. }
$$

4.2. Fractional Laplacians $-\alpha$-stable processes. Our result also applies to various classes of jump processes including $\alpha$-stable processes. Dirichlet forms of such processes have attracted tremendous attention in various respects in recent years, including study of eigenvalue estimates [18, 20] and heat kernel estimates and estimates for solutions of the corresponding partial differential equations [1, 2, 9, 26, 27].

Let us first present the basic model. Let $X=\mathbb{R}^{d}$ and $\Delta$ be the usual Laplacian on $L^{2}\left(\mathbb{R}^{d}\right)$. The fractional Laplacian $\mathcal{L}_{\alpha}=(-\Delta)^{\alpha / 2}$ is the infinitesimal generator of a Markov process known as $\alpha$-stable process for $0<\alpha<2$. The corresponding Dirichlet form is (up to a constant) defined by

$$
\mathcal{E}_{\alpha}(u, v):=\int_{X \times X \backslash D}(u(x)-u(y))(v(x)-v(y))|x-y|^{-d-\alpha} \mathrm{d} x \mathrm{~d} y,
$$




$$
\mathcal{D}_{\alpha}:=\left\{u \in L^{2}(X): \mathcal{E}_{\alpha}(u)<\infty\right\},
$$

where $D:=\{(x, x): x \in X\} \subset X \times X$ denotes the diagonal.

This is obviously a non-local Dirichlet form. It is regular since by basic theory of Sobolev spaces the test functions are a core for this Dirichlet form. Now, clearly a power of $\mathcal{L}_{\alpha}$, viz $-\Delta$, is spatially locally compact (see Section 4.1) and so is then $\mathcal{L}_{\alpha}$ itself, by [40], Thm 1.3. Hence, both the decomposition principle and the Persson theorem of the previous section apply to $\mathcal{L}_{\alpha}$.

As mentioned in the introduction, this seems to be the first result on a decomposition principle for an operator that is neither a Laplacian on a manifold nor on a graph.

More generally, consider $U \subset \mathbb{R}^{d}$ open

$$
\mathcal{E}(u, v):=\int_{U \times U \backslash D}(u(x)-u(y))(v(x)-v(y)) j(x, y) \mathrm{d} x \mathrm{~d} y
$$

on

$$
\mathcal{D}_{0}:=\left\{u \in C_{c}(U): \mathcal{E}(u)<\infty\right\},
$$

with a symmetric positive weight function $j$. This defines a closable form and we denote by $\mathcal{D}$ the domain of its closure and by $\mathcal{L}$ the corresponding non-negative operator.

Corollary 4.3. Assume that $j, \mathcal{E}, \mathcal{L}$ are as above and that additionally:

(i) $\mathcal{D}_{0}$ is dense in $C_{c}(U)$ with respect to $\|\cdot\|_{\infty}$.

(ii) For some $\alpha \in(0,2), c>0$ :

$$
j(x, y) \geqslant c|x-y|^{-d-\alpha} \text { for all } x, y \in \mathbb{R}^{d} .
$$

Then

(1) $\mathcal{L}$ is spatially locally compact.

(2) For every compact subset $K \subset U$ :

$$
\sigma_{\text {ess }}(\mathcal{L})=\sigma_{\text {ess }}\left(\mathcal{L}_{U \backslash K}\right) .
$$

(3) Perssons' theorem holds:

$$
\inf \sigma_{e s s}(\mathcal{L})=\sup _{K} \inf \sigma\left(\mathcal{L}_{U \backslash K}\right),
$$

where the supremum is taken over the net of compact subsets of $U$.

The proof is evident: by assumption it follows that the form domain of $\mathcal{L}$ embeds into the form domain of $\mathcal{L}_{\alpha}$. Hence, $\mathcal{L}$ is spatially locally compact by Remark 2.2 (b) above so that we can apply our Theorems 2.3 and 3.2 . Evidently, as well, if $j$ satisfies an upper bound of the form as in (ii) above, possibly with a different $\alpha^{\prime}$ then the denseness assumption (i) is satisfied. Thus our analysis includes large classes of examples, studied, e.g. in [1, 17]; see also the references there for more pointers to the literature. We should also like to point out that under such stronger conditions, heat kernel bounds are known which would give an alternative, more complicated way of showing that the associated operator is spatially locally compact.

Of course, we can consider the more general case of fractional Laplacians on manifolds or even jump type Dirichlet forms on metric measure spaces whenever Gaussian estimates or - sufficient for our purpose - just ultracontractivity estimates are available. See [2, 10, 27, 30] for a start. 
4.3. Regular Dirichlet forms on discrete spaces. The study of Laplacians on graphs has a long history (see, e.g., the monographs [11, 13] and the references therein). Much research has been devoted to study graphs with uniformly bounded vertex degree. In recent years, various issues related to unboundedness of the vertex degree have been studied. A glimpse of these developments can be inferred from the survey articles [37, 34] and references therein. The mentioned issues can be studied in various settings. The most general setting seems to be the one introduced in [36, which we now recall: Let $X$ be a countable set. Let $m$ be a measure on $X$ with full support, i.e., $m$ is a map on $X$ taking values in $(0, \infty)$. A symmetric weighted graph over $X$ or graph for short is a pair $(b, c)$ consisting of a map $c: X \rightarrow[0, \infty)$ and a map $b: X \times X \rightarrow[0, \infty)$ with $b(x, x)=0$ for all $x \in X$ satisfying the following two properties:

(b1) $b(x, y)=b(y, x)$ for all $x, y \in X$.

(b2) $\sum_{y \in X} b(x, y)<\infty$ for all $x \in X$.

To $(b, c)$ we associate the form $\mathcal{E}_{b, c}^{\text {comp }}$ defined on the set $C_{c}(X)$ of functions on $X$ with finite support by

$$
\begin{gathered}
\mathcal{E}_{b, c}^{\mathrm{comp}}: C_{c}(X) \times C_{c}(X) \longrightarrow[0, \infty) \\
\mathcal{E}_{b, c}^{\mathrm{comp}}(u, v)=\frac{1}{2} \sum_{x, y \in X} b(x, y)(u(x)-u(y)) \overline{(v(x)-v(y))}+\sum_{x} c(x) u(x) \overline{v(x)} .
\end{gathered}
$$

Observe that the first sum is convergent by properties (b1) and (b2); the second sum is finite. The form $\mathcal{E}_{b, c}^{\text {comp }}$ is closable in $\ell^{2}(X, m)$; the closure will be denoted by $\mathcal{E}_{b, c, m}$. Now, the forms $\mathcal{E}_{b, c, m}$ for $(b, c)$ graph over $X$ are exactly the regular Dirichlet forms on $\ell^{2}(X, m)$ ([36, 23]). Clearly, this setting contains both combinatorial graphs as well as weighted graphs with uniformly bounded vertex degree. From our main theorems, we then obtain the following consequence.

Corollary 4.4. Let $\mathcal{E}_{b, c, m}$ be as above with the additional assumption that $m$ is bounded below by a positive constant. Then the generator $\mathcal{L}_{b, c, m}$ is spatially locally compact. In particular, the decomposition principle, Theorem 2.3 and Persson's theorem, Theorem 3.2 above apply.

As for the proof we note that spatial local compactness is an evident consequence of the fact that $\mathbb{1}_{E}$ projects onto a finite dimensional space for any set $E$ of finite measure.

The corollary contains and complements various earlier results: In 35] the case of $c=0, b$ taking values in $\{0,1\}$ only and $m \equiv 1$ is covered (see [21] for corresponding earlier results dealing with the normalized Laplacian). The reference [37] states that a Persson theorem holds for graphs once a decomposition principle is known and notes that a decomposition principle holds for locally finite graphs.

\section{SCHRÖDINGER TYPE OPERATORS - MEASURE PERTURBATIONS}

We can carry over the ideas presented above to also treat Schrödinger operators i.e. measure perturbations of Dirichlet forms by invoking the methods provided in [59]. Details are discussed next. We note in passing that quite 
general potentials have also been discussed in [3], where a Persson type theorem is presented, restricted, however to the case of perturbations of the classical Laplacian on Euclidean space.

From [23], we infer that every $u \in \mathcal{D}$ admits a quasi-continuous version $\tilde{u}$, the latter being unique up to sets of capacity zero. This allows us to consider measure potentials in the following way: see [44], [45] for the special case of the Laplacian and locally finite measures, 55] and the references in there. Let $\mathcal{M}_{0}=\{\mu: \mathcal{B} \rightarrow[0, \infty] \mid \mu$ a measure $\mu \ll$ cap $\}$, where $\ll$ denotes absolute continuity, i.e. the property that $\mu(B)=0$ whenever $B \in \mathcal{B}$ and $\operatorname{cap}(B)=0$. For measures in $\mathcal{M}_{0}$ we explicitly allow that the measure takes the value $\infty$. A particular example is $\infty_{B}$, defined by

$$
\infty_{B}(E)=\infty \cdot \operatorname{cap}(B \cap E),
$$

with the convention $\infty \cdot 0=0$. Note that for $\mu \in \mathcal{M}_{0}$ we have that $\mu[u, v]:=$ $\int_{X} \tilde{u} \tilde{v} \mathrm{~d} \mu$ is well defined for $u, v \in \mathcal{D}(\mu)$, where $\mathcal{D}(\mu)=\{u \in \mathcal{D} \mid \tilde{u} \in$ $\left.L^{2}(X, \mathcal{B}, \mu)\right\}$. It is easy to see that

$$
\mathcal{D}(\mathcal{E}+\mu):=\mathcal{D} \cap \mathcal{D}(\mu),(\mathcal{E}+\mu)[u, v]:=\mathcal{E}[u, v]+\mu[u, v]
$$

gives a closed form (not necessarily densely defined). One can check that, e.g., $\mathcal{E}+\infty_{B}=\left.\mathcal{E}\right|_{\mathcal{D}_{0}\left(B^{c}\right)}$, where $\mathcal{D}_{0}(U)=\left\{u \in \mathcal{D}|\tilde{u}|_{U^{c}}=0\right.$ q.e. $\}$ and that we get

$$
\mathcal{E}+\infty_{B}=\mathcal{E}_{U}
$$

provided $U:=B^{c}$ is open; see the discussion in Section 1 above.

If $\mu^{-} \in \mathcal{M}_{0}$ is form small w.r.t. $\mathcal{E}+\mu^{+}$, we can furthermore define $\mathcal{E}+$ $\mu=\mathcal{E}+\mu^{+}-\mu^{-}$by the KLMN-theorem, [50], Theorem X.17 and denote the associated selfadjoint operator (which might be selfadjoint in a smaller Hilbert space!) by $\mathcal{L}+\mu$. Note that for $\mu^{+}=0$ this form boundedness implies that $\mu^{-}$is a Radon measure, i.e., finite on all compact sets.

We now discuss, whether the results from Theorems 2.3 and 3.2 remain valid for $\mathcal{L}+\mu$. First note that by the results from [41], the spatial local compactness property carries over from $\mathcal{L}$ to $\mathcal{L}+\mu$, since we can apply [41], Theorem 1.3, noting that in the notation of the latter paper $Q(\mathcal{L}+\mu)=$ $\mathcal{D}(\mathcal{E}+\mu) \subset Q(\mathcal{L})=\mathcal{D}$ with continuous embedding.

Thus, the extension of our results to Schrödinger type operators $\mathcal{L}+\mu$ essentially boils down to checking, whether Proposition 2.4 and Corollary 2.5 hold.

Following [59], we now introduce the appropriate Kato condition, so let $\hat{\mathcal{S}}_{K}$ and $c_{\alpha}(\mu)$ be defined as in the latter paper, with respect to $\mathcal{L}$ (called $H$ in that latter reference).

We first summarize the approximation results from [59], using, that due to our separability assumption here, we can work with sequences instead of nets.

Lemma 5.1. Let $\mathcal{E}$ be a regular Dirichlet form on $L^{2}(X, m)$ with domain $\mathcal{D}$ and associated selfadjoint operator $\mathcal{L}, \mu_{+} \in \mathcal{M}_{0}$ and $\mu_{-} \in \hat{\mathcal{S}}_{K}$ with $c_{\alpha}\left(\mu_{-}\right)<$ 1 for some $\alpha>0$. Then $\mu_{-}$is form small w.r.t. $\mathcal{L}$.

(1) Then $\mu_{-}$is form small w.r.t. $\mathcal{L}$.

(2) There is a sequence $\left(V_{n}\right)$ in $L^{\infty}(X, m)_{+}$such that

$$
\mathcal{L}-\mu_{-}+V_{n} \rightarrow \mathcal{L}+\mu \text { as } n \rightarrow \infty
$$


in the strong resolvent sense.

(3) There is a sequence $\left(W_{n}\right)$ in $L^{\infty}(X, m)_{+}$such that,

$$
c_{\alpha}\left(W_{n}\right) \leqslant c_{\alpha}\left(\mu_{-}\right) \text {for all } n \in \mathbb{N} \text {. }
$$

and

$$
\mathcal{L}-W_{n} \rightarrow \mathcal{L}-\mu_{-} \text {as } n \rightarrow \infty
$$

in the strong resolvent sense.

(4) For the semigroup differences we get the pointwise estimates

$$
\left|\left(e^{-t(\mathcal{L}+\mu)}-e^{-t\left(\mathcal{L}_{G}+\mu\right)}\right) f(x)\right| \leqslant\left(e^{-t\left(\mathcal{L}-\mu_{-}\right)}-\left(e^{-t\left(\mathcal{L}_{G}-\mu_{-}\right)}|f|(x) .\right.\right.
$$

(5) $e^{-t(\mathcal{L}+\mu)}$ maps from $L^{1}$ to $L^{1}$ provided $c_{\alpha}\left(\mu_{-}\right)<1$ for some $\alpha>0$.

Proof. (1) follows from [59], Thm. 3.1.

For (2) we can use the arguments from the proof of Theorem 6.1, part (ii) in the latter reference using, as mentioned above, that an increasing sequence of compact sets can be used instead of a net.

(3) follows from [59], Thm. 3.5, where again, we can choose a sequence instead of a net.

(4) follows from (2) and the Feynman-Kac formula, respectively the Trotter product formula.

(5) is a consequence of [59], Thm. 3.3.

Note that (4) in the above lemma is reminiscent of results in [31]. We are now in position to generalize 2.4 to Schrödinger type operators.

Proposition 5.2. Let $\mathcal{E}, \mathcal{L}$ be as above, $\mu_{+} \in \mathcal{M}_{0}$ and $\mu_{-} \in \hat{\mathcal{S}}_{K}$ with $c_{\alpha}\left(\mu_{-}\right)<\frac{1}{2}$ for some $\alpha>0$. Then there exists $C$, depending on $\alpha>$ $0, c_{\alpha}\left(\mu_{-}\right), t>0$ such that the following holds: for $B \subset X$ a closed set of finite capacity and $G:=X \backslash B$, for measurable $A \subset X$ and $t>0$ we have

$$
\begin{aligned}
\left\|\mathbb{1}_{A}\left(e^{-t(\mathcal{L}+\mu)}-e^{-t\left(\mathcal{L}_{G}+\mu\right)}\right)\right\| & \leqslant C \cdot \sup _{x \in A}\left[\mathbb{P}^{x}\left\{\omega \mid \sigma_{B}(\omega) \leqslant t\right\}\right]^{\frac{1}{2}} \\
& \leqslant C \cdot \sup _{x \in A} e^{\frac{t}{2}}\left[e_{B}(x)\right]^{\frac{1}{2}}
\end{aligned}
$$

Proof. First of all, Part (4) from the preceding lemma shows that we can assume that $\mu_{+}=0$. By Part (3) we can replace the negative part of $\mu$ by a bounded function that satisfies the same Kato condition, call it $W$. Therefore, we are left to estimate $\left\|\mathbb{1}_{A}\left(e^{-t(\mathcal{L}-W)}-e^{-t\left(\mathcal{L}_{G}-W\right)}\right)\right\|$ :

for any $f \in L^{2}$ and a.e. $x \in X$ :

$$
\begin{aligned}
\left|\left(e^{-t(\mathcal{L}-W)}-e^{-t\left(\mathcal{L}_{G}-W\right)}\right) f(x)\right| & \left.=\mid \mathbb{E}^{x}\left[f \circ X_{t} e^{-\int_{0}^{t} W \circ X_{s} d s}\left(1-\mathbb{1}_{\left\{\omega \mid \sigma_{B}(\omega)>t\right.}\right\}\right)\right] \mid \\
& =\left|\mathbb{E}^{x}\left[f \circ X_{t} e^{-\int_{0}^{t} W \circ X_{s} d s} \mathbb{1}_{\left\{\omega \mid \sigma_{B}(\omega) \leqslant t\right\}}\right]\right| \\
& \leqslant \mathbb{E}^{x}\left[|f|^{2} \circ X_{t} \cdot e^{-2 \int_{0}^{t} W \circ X_{s} d s}\right]^{\frac{1}{2}} \mathbb{P}^{x}\left\{\omega \mid \sigma_{B}(\omega) \leqslant t\right\}^{\frac{1}{2}}
\end{aligned}
$$

by Cauchy-Schwarz. Note that the integral appearing in the first factor gives

$$
\mathbb{E}^{x}\left[|f|^{2} \circ X_{t} e^{-2 \int_{0}^{t} W \circ X_{s} d s}\right]=e^{-t(\mathcal{L}-2 W)}\left(|f|^{2}\right)(x)
$$


an integrable function of $x$, with integral bounded by $e\left\|^{-t(\mathcal{L}-2 W)}\right\|_{1,1}\|f\|_{2}^{2}$. This is finite as the semigroup maps $L^{1}$ to itself by [59], Thm. 3.3 or part (5) of the preceding Lemma, where we use that $c_{\alpha}(2 W)=2 c_{\alpha}(W)$. Moreover the norm from $L^{1}$ to $L^{1}$, indicated by the subscript 1,1 above can be estimated in terms of the given quantities. This gives the asserted estimate just like in the proof of Proposition 2.4 above.

The remaining steps in the proof of our main results can be easily adapted as well to give:

Theorem 5.3. Let $\mathcal{E}$ be a regular Dirichlet form on $L^{2}(X, m)$ with associated selfadjoint operator $\mathcal{L}$ that is spatially locally compact. Let $\mu_{+} \in \mathcal{M}_{0}$ and $\mu_{-} \in \hat{\mathcal{S}}_{K}$ with $c_{\alpha}\left(\mu_{-}\right)<\frac{1}{2}$ for some $\alpha>0$.

(1) Let $B \subset X$ be a closed set of finite capacity and denote $G:=X \backslash B$. Then, the operator $\varphi\left(\mathcal{L}_{G}\right)-\varphi(\mathcal{L})$ is compact for every $\varphi \in C_{0}(\mathbb{R})$. In particular,

$$
\sigma_{\text {ess }}\left(\mathcal{L}_{G}+\mu\right)=\sigma_{\text {ess }}(\mathcal{L}+\mu)
$$

holds.

(2)

$$
\inf \sigma_{e s s}(\mathcal{L}+\mu)=\lim _{K \rightarrow X} \inf \sigma\left(\mathcal{L}_{X \backslash K}+\mu\right)
$$

\section{ACKNOWLEDGMENTS}

D.L. gratefully acknowledges many inspiring discussions with Matthias Keller on a wide range of topics related to the present paper as well as partial support form German Research Foundation (DFG).

\section{REFERENCES}

[1] M. Barlow, R. Bass, Z. Q. Chen and M. Kassmann, Non-local Dirichlet forms and symmetric jump processes. Trans. Amer. Math. Soc., 361(4), (2009), 1963-1999.

[2] M. Barlow, A. Grigor' yan, T. Kumagai, Heat kernel upper bounds for jump processes and the first exit time. J. Reine Angew. Math. 626 (2009), 135-157.

[3] I. Beltiţă , H. Cornean, On a theorem of Arne Persson, Cubo 6 (2004), 1-14.

[4] M. Biroli, U. Mosco, A Saint-Venant type principle for Dirichlet forms on discontinuous media. Ann. Mat. Pura Appl. (4) 169 (1995), 125-181

[5] M. Biroli, N. A. Tchou, Asymptotic behaviour of relaxed Dirichlet problems involving a Dirichlet-Poincaré form, Z. Anal. Anwendungen 16 (1997), 281-309

[6] N. Bouleau, F. Hirsch, Dirichlet forms and analysis on Wiener space, Vol. 14 of de Gruyter Studies in Mathematics, Walter de Gruyter \& Co., Berlin, 1991.

[7] A. Boutet de Monvel, D. Lenz, P. Stollmann, Sch'nol's theorem for strongly local forms, Israel J. Math. 173 (2009), 189-211.

[8] A. Boutet de Monvel, P. Stollmann, Eigenfunction expansions for generators of Dirichlet forms, J. Reine Angew. Math. 561 (2003), 131-144.

[9] Z.-Q. Chen, T. Kumagai, Heat kernel estimates for stable-like processes on d-sets, Stochastic Process. Appl. 108 (2003), 27-62.

[10] Z.-Q. Chen, T. Kumagai, Heat kernel estimates for jump processes of mixed types on metric measure spaces. Probab. Theory Rel. Fields 140 (2008), 277-317.

[11] F. R. K. Chung, Spectral graph theory, CBMS Regional Conference Series in Mathematics, vol. 92, American Mathematical Society, Providence, RI, 1997. 
[12] F. R. K. Chung, A. Grigor'yan, S.-T. Yau, Higher eigenvalues and isoperimetric inequalities on Riemannian manifolds and graphs, Comm. Anal. Geom. 8 (2000), 969-1026.

[13] Y. Colin de Verdière, Spectres de graphes, Cours Spécialisés, vol. 4., Société Mathématique de France, Paris, 1998.

[14] E. B. Davies, Spectral theory and differential operators, Vol. 42 of Cambridge Studies in Advanced Mathematics, Cambridge University Press, Cambridge, 1995.

[15] M. Demuth, P. Stollmann, G. Stolz, and J. van Casteren, Trace norm estimates for products of integral operators and diffusion semigroups, Integral Equations Operator Theory 23(2), 145-153 (1995).

[16] H. Donnelly, P. Li, Pure point spectrum and negative curvature for noncompact manifolds, Duke Math. J. 46 (1979), 497-503.

[17] M. Felsinger, M. Kassmann, and P. Voigt, The Dirichlet problem for nonlocal operators, Math. Z. 279,3-4 (2015), 779-809.

[18] R. Frank, L. Geisinger, Refined semiclassical asymptotics for fractional powers of the Laplace operator. J. Reine Angew. Math. 712 (2016), 1-37.

[19] R. Frank, D. Lenz, D. Wingert, Intrinsic metrics for non-local symmetric Dirichlet forms and applications to spectral theory. J. Funct. Anal. 266 (2014), 4765-4808.

[20] R. Frank, E. Lenzmann, L. Silvestre, Uniqueness of radial solutions for the fractional Laplacian. Comm. Pure Appl. Math. 69 (2016), 1671 - 1726.

[21] K. Fujiwara, Laplacians on rapidly branching trees. Duke Math. J. 83 (1996), 191202.

[22] M. Fukushima, Dirichlet forms and symmetric Markov processes, North-Holland, Amsterdam, 1980.

[23] M. Fukushima, Y. Ōshima, M. Takeda, Dirichlet forms and symmetric Markov processes, Vol. 19 of de Gruyter Studies in Mathematics, Walter de Gruyter \& Co., Berlin, 1994.

[24] A. Grigor'yan, Estimates of heat kernels on Riemannian manifolds, in: Spectral theory and geometry (Edinburgh, 1998), London Math. Soc. Lecture Note Ser., vol 273, 140-225, Cambridge Univ. Press, Cambridge, 1999

[25] A. Grigor' yan, Heat kernel and analysis on manifolds. AMS/IP Studies in Advanced Mathematics, vol 47, American Mathematical Society, Providence, RI, 2009,

[26] A. Grigor'yan, E. Hu, J. Hu, Lower estimates of heat kernels for non-local Dirichlet forms on metric measure spaces, J. Funct. Anal. 272 (2017), 3311-3346.

[27] A. Grigor'yan, J. Hu, and K.-S. Lau, Heat kernels on metric measure spaces and an application to semilinear elliptic equations, Trans. Amer. Math. Soc. 355(5) (2003), 2065-2095.

[28] G. Grillo, On Persson's Theorem in local Dirichlet spaces, Zeitschrift für Analysis and ihre Anwendungen 7 (1998), 329-338.

[29] S. Haeseler, M. Keller, R. Wojciechowski, Volume growth and bounds for the essential spectrum for Dirichlet forms. J. Lond. Math. Soc. (2) 88 (2013), 883-898.

[30] J. Hu, T. Kumagai, Nash-type inequalities and heat kernels for non-local Dirichlet forms. Kyushu J. Math. 60 (2006), no. 2, 245-265.

[31] D. Hundertmark, B. Simon, A diamagnetic inequality for semigroup differences. J. Reine Angew. Math. 571 (2004), 107-130.

[32] N. Jacob, R. L. Schilling, Towards an $L^{p}$ Potential Theory for Sub-Markovian Semigroups: Kernels and Capacities, Acta Mathematica Sinica, English Series, 22 (2006), $1227-1250$.

[33] T. Kato, Perturbation theory for linear operators, Die Grundlehren der mathematischen Wissenschaften, Band 132, Springer-Verlag New York, Inc., New York, 1966.

[34] M. Keller, Intrinsic metrics on graphs - A survey to appear in: Mathematical Technology of Networks (Proc. Bielefeld 2013), Proc. Math. \& Stat. Springer-Verlag, New York.

[35] M. Keller, The essential spectrum of the Laplacian on rapidly branching tessellations. Math. Ann. 346 (2010), 51-66.

[36] M. Keller, D. Lenz, Dirichlet forms and stochastic completeness of graphs and subgraphs, J. reine angew. Math. 666 (2012), 189-223. 
[37] M. Keller, D. Lenz, Unbounded Laplacians on graphs: basic spectral properties and the heat equation, Math. Model. Nat. Phenom. 5 (2010), 198-224.

[38] M. Keller, D. Lenz, H. Vogt, R. Wojciechowski, Note on basic features of large time behaviour of heat kernels, to appear in: J. Reine Angew. Math. 708 (2015), 73-95.

[39] D. Lenz, C. Schubert, and P. Stollmann, Eigenfunction expansions for SchrÖdinger operators on metric graphs, Integral Equations Operator Theory 62 (2008), 541-553.

[40] D. Lenz, P. Stollmann, D. Wingert, Compactness of Schrödinger semigroups. Math. Nachr. 283 (2010), 94-103.

[41] D. Lenz, P. Stollmann, I. Veselić, The Allegretto-Piepenbrink theorem for strongly local Dirichlet forms, Doc. Math. 14 (2009) 167-189.

[42] P. Li and S. T. Yau, on the parabolic kernel of the Schrödinger operator. Acta Math., 156(3-4): 153-201, 1986.

[43] Z. M. Ma, M. Röckner, Introduction to the theory of (nonsymmetric) Dirichlet forms, Universitext, Springer-Verlag, Berlin, 1992.

[44] V.G. Maz'ja, On the theory of the higher-dimensional Schrödinger operator, Izv. Akad. Nauk SSSR Ser. Mat. 28 (1964), 1145-1172.

[45] V. G. Maz'ja, Sobolev spaces, Springer Series in Soviet Mathematics (Springer-Verlag, Berlin, 1985), Translated from the Russian by T. O. Shaposhnikova.

[46] V. Maz'ya, M. Shubin, Discreteness of spectrum and positivity criteria for Schrödinger operators, Ann. of Math. (2) 162 (2005), 919-942.

[47] A. Molchanov, On the discreteness of the spectrum conditions for selfadjoint differential equations of the second order, Trudy Moskv. Mat. Obshch. 2 (1953), 169-199.

[48] A. Persson, Bounds for the discrete part of a semibounded Schrödinger operator, Math. Scand. 8 (1960), 143-153.

[49] M. Reed, B. Simon, Methods of modern mathematical physics. I. Functional analysis, Academic Press, New York, 1972.

[50] M. Reed and B. Simon, Methods of modern mathematical physics. II. Fourier analysis, self-adjointness (Academic Press [Harcourt Brace Jovanovich Publishers], New York, 1975).

[51] M. Reed and B. Simon, Methods of modern mathematical physics. IV. Analysis of operators (Academic Press [Harcourt Brace Jovanovich Publishers], New York, 1978).

[52] C. Rose, Heat kernel upper bound on Riemannian manifolds with locally uniform Ricci curvature integral bounds, Journal of Geometric Analysis, 1-14, 2016.

[53] B. Simon, Schrödinger semigroups. Bull. Amer. Math. Soc. (N.S.) 7 (1982), 447-526.

[54] P. Stollmann, Smooth perturbations of regular Dirichlet forms, Proc. Amer. Math. Soc. 116 (1992), 747-752.

[55] P. Stollmann, Scattering by obstacles of finite capacity, J. Funct. Anal. 121 (1994), 416-425.

[56] P. Stollmann, Störungstheorie von Dirichletformen mit Anwendungen auf Schrödingeroperatoren, Habilitationsschrift, Universität Frankfurt, 1994.

[57] P. Stollmann, A convergence theorem for Dirichlet forms with applications to boundary value problems with varying domains. Math. Z. 219 (1995), 275-287.

[58] P. Stollmann, A dual characterization of length spaces with application to Dirichlet metric spaces, Studia Math. 198 (2010), 221-233.

[59] P. Stollmann, J. Voigt, Perturbation of Dirichlet forms by measures. Potential Anal. 5 (1996), 109-138.

[60] K.-T. Sturm, Analysis on local Dirichlet spaces. I. Recurrence, conservativeness and $L^{p}$-Liouville properties, J. Reine Angew. Math. 456 (1994), 173-196.

[61] . K.-T. Sturm, On the Geometry Defined by Dirichlet Forms, Progress in Probability 36 (1995), 231-242.

[62] H. Weyl, Über gewöhnliche Differentialgleichungen mit Singularitäten und die zugehörigen Entwicklungen willkürlicher Funktionen, Math. Ann. 68 (1910), 220-269.

[63] H. Weyl, Über beschränkte quadratische Formen, deren Differenz vollstetig ist. Rend. Circ. Mat. Palermo 27 (1909), 220-269.

${ }^{1}$ Mathematisches Institut, Friedrich Schiller Universität Jena, D-03477 Jena, GERMANY, DANIEL.LENZ@UNI-JENA.DE, URL: HTTP://WWW.ANALYSIS-LENZ.UNI-JENA.DE/ 
${ }^{2}$ Fakultät für Mathematik, TU Chemnitz, 09107 Chemnitz, Germany 